\title{
The dominance of secondary nuclei in the cosmic radiation and the modulation of the nuclear species at the injection of the galactic accelerator
}

\author{
Antonio Codino* \\ University of Perugia and INFN \\ E-mail: antonio.codino@pg.infn.it
}

\begin{abstract}
The cosmic ray abundances are compared to those of the quiescent matter referred to as galactic or solar abundances. Cosmic-ray and Galactic abundances are normalized to Iron. The comparison takes advantage of the recent data of the energy spectra of the cosmic nuclei in the interval $5 \times$ $10^{11}-5 \times 10^{14} \mathrm{eV}$ and the observation of a constant spectral index of $2.67 \pm 0.05$. The ratio of cosmic-ray to galactic abundances conforms to a remarkable regularity : the odd nucleus has a greater ratio than the adjacent even nucleus for the 12 nuclear species in the range, $1 \leq \mathrm{Z} \leq$ 28 being $\mathrm{Z}$ the atomic number. Since many decades this rule is explained by the production of secondary nuclei by spallation reactions of primary nuclei in the interstellar medium. From this regularity it is inferred that the mechanism accelerating cosmic rays (galactic accelerator) does not alter the quiescent matter abundances. From similar empirical arguments it is argued that primary proton and primary helium in cosmic rays are highly depleted at the injection stage of the galactic accelerator. The depletion of $\mathrm{H}$ and $\mathrm{He}$ cosmic nuclei relative to Iron amounts to suppression factors higher than $10^{3}-10^{4}$ which outnumber those previously reported in the past and recent literature.
\end{abstract}

The 34th International Cosmic Ray Conference,

30 July- 6 August, 2015

The Hague, The Netherlands

${ }^{*}$ Speaker. 


\section{Introduction}

Eighteen orders of magnitudes separate the total mass of the cosmic rays from that of the Milky Way Galaxy. If this enormous factor is suppressed making to coincide the relative amounts of the atoms of the two matter distributions immediately emerge remarkable similarities and striking differences. These features have been debated since many decades in the literature. Presently one major aspect to analyze the two distributions is the identification of the mechanism accelerating cosmic rays in the Galaxy. Whatever might be this mechanism, the resemblance and the deviations resulting from the comparison of the two distributions, have to be quantitatively accounted for.

The theoretical pattern behind this paper is unbiased and it assumes a cosmic-ray life cycle subdivided into the injection phase, the acceleration process and the extinction of cosmic rays by any mechanisms. For example, a well assessed mechanism for extinction is the disappearence of low-energy cosmic rays in the solar wind. It is also assumed that the acceleration process do not modify the chemical composition present at the injection stage. Galactic regions where quiescent matter suffers some filter before acceleration are regarded as sources. The undifferentiated interstellar medium could be regarded as cosmic-ray sources, a simple notion from Leaky Box Models. The focus of this paper is on this filtering process.

As customary the atomic number of the nucleus is denoted by $\mathrm{Z}$ and the atomic weight by $\mathrm{A}$. The present analysis encompasses the range, $1 \leq \mathrm{Z} \leq 28$ populated by the most abundant elements and explored by numerous cosmic-ray experiments.

Cosmic-ray abundances are given in total kinetic energy per particle, and not in $\mathrm{GeV}$ per nucleon. High energy experiments above the $3 \times 10^{15} \mathrm{eV}$ use this unit of measurement. Recently the TRACER experiment [1], that collected data below $10^{15} \mathrm{eV}$ measuring fluxes of 10 individual nuclear species, expressed the results in this unit. Notice however, the adoption of this unit has consequences.

\section{Abundances of elements in the local interstellar medium}

Measurements of the relative amounts of elements, hereafter denoted by $A_{g a}(Z)$, in the range $1 \leq \mathrm{Z} \leq 28$ come from observations of the solar atmosphere, meteorites intercepted by the Earth and substructures of the Galaxy such as B stars, HII regions, planetary nebulae and others. For a number of empirical reasons atmospheric solar abundances are representative of those of the local interstellar medium. In principle solar abundances differ from Galactic abundances and the term quiescent matter abundances is used here to represent the undifferentiated interstellar medium. Since cosmic-ray matter is extremely energetic it is clearly distinguished from any other forms of matter aggregations, like for example the cold molecular clouds or the atmospheres of hot stars, globally denoted here as quiescent matter. Cosmic rays have huge amounts of kinetic energy while quiescent matter has insignificant amounts compared to the rest mass of nuclei and electrons.

A recent compilation of measurements of atmospheric solar abundances is reported and discussed by others [2]. The matter abundances of this compilation are in Fig. 1. The data of Fig. 1 are normalized to Iron while those in the original work [3] to Hydrogen. According to the data of Fig. 1 the most abundant element is Hydrogen whereas the rarest one is Lithium. In a previous compilation Berillium, instead of Lithium, resulted the rarest atom. 


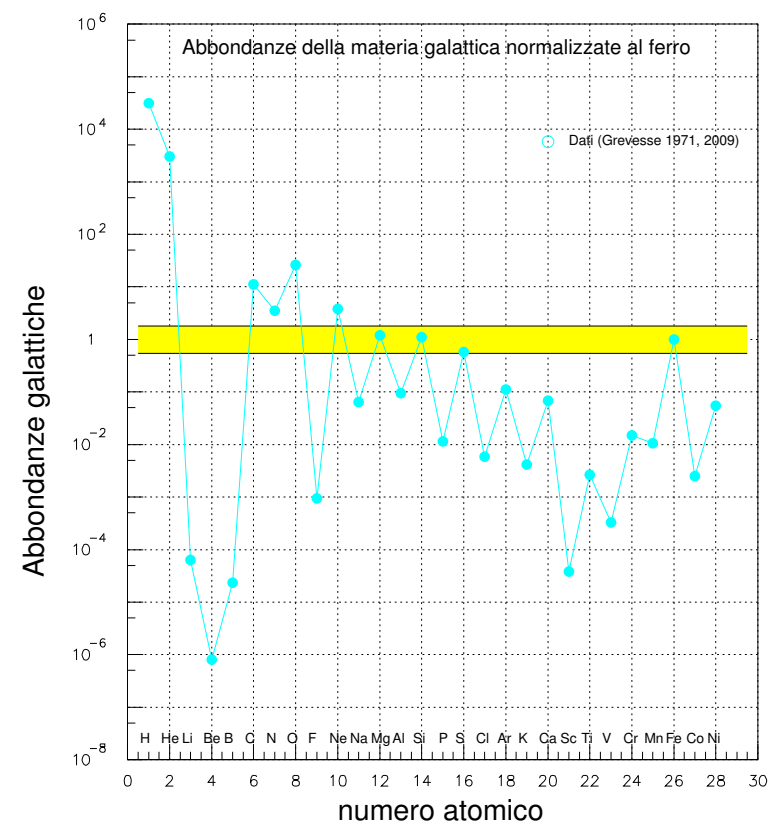

Figure 1: Abundances of 28 elements of the quiescent matter from Hydrogen to Nickel normalized to that of Iron. These abundances are representatives of the interstellar medium and star atmospheres. The yellow strip is only a visual guide around the unitary value of the Fe nucleus within a factor \pm 2 .

A verification of atmospheric solar abundances of Fig. 1 is the comparison with the meteoritic abundances. The two distributions coincide except for volatile elements such as $\mathrm{He}, \mathrm{N}, \mathrm{C}, \mathrm{Ne}, \mathrm{O}$ and Ar.

Believing to modern Cosmology, $\mathrm{H}$ and $\mathrm{He}$ are abundant primordial elements while $\mathrm{Li}$, $\mathrm{Be}$ and $\mathrm{B}$ are very scarce primordial ones. Elements heavier than Boron are produced in the star interiors via nuclear reactions. Light particles such as Deuterium (hereafter $D$ ), $\mathrm{Li}, \mathrm{Be}$ and $\mathrm{B}$ are burned at the very initial stages of the star lifetimes and hence their paucity is expected even to increase if the galactic production were insignificant.

Nuclei in Fig. 1 in the range, $5 \leq Z \leq 28$ conform with the Even-Odd Effect : the nucleus with an even $\mathrm{Z}$ is more abundant of the adjacent (Z-1) nucleus. Twelve pairs of nuclei obey this empirical rule which is ultimately due to the stability of the quantum mechanical edifice of even nuclei compared to that, more fragile, of odd nuclei. Nuclei in the range, $1 \leq \mathrm{Z} \leq 4$ are believed to be primordial and abundance hierarchy depends on the cosmological model which however is not of critical importance in this work.

\section{Elemental abundances in the cosmic radiation}

In the energy interval $10^{10}-10^{15} \mathrm{eV}$ all processes forging the features of cosmic rays are at regime because the energy spectra of individual nuclei have equal spectral indices of 2.67 within the error bars of \pm 5 [1] . Consequently, cosmic rays preserve exactly the same abundances in the 




Figure 2: Cosmic-ray abundances of 28 nuclei normalized to the Iron flux at $10^{12} \mathrm{eV}$. Data are expressed in total kinetic energy per nucleus and are provided by balloon-borne experiments (blue triangles, Tracer; red triangles, Cream). Fluxes of the compilations (green dots) $[5,6]$ are extrapolated at $10^{12} \mathrm{eV}$.

more than 5 energy decades. This energy band, characterized by the universal index $\gamma_{u}=2.67$, is adequate, almost ideal, for flux normalization. Thus cosmic-ray features in the band $10^{10}-10^{15} \mathrm{eV}$ are insensitive to both solar modulation effects and propagation effects, those causing the knees of individual nuclei above $10^{15} \mathrm{eV}$.

Cosmic-ray abundances in the range $1 \leq \mathrm{Z} \leq 28$ are shown in Fig. 2. All fluxes are normalized to the Iron flux of $3.39 \times 10^{16}$ particles $/\left(m^{2} s s r \mathrm{eV}^{1.5}\right)$ at $10^{12} \mathrm{eV}$. Besides Tracer data [1] (blu triangles) and those from Cream experiment (red triangles) [4] two data compilations at various energies [5] and at $10^{14} \mathrm{eV}$ (see Fig. 17 of ref [6] ) extrapolated to $10^{12} \mathrm{eV}$ are reported in Fig. 2. Cosmic-ray abundances or fluxes are denoted here by $F_{c r}(Z)$. Flux normalization to another energy value, within $10^{10}-10^{15} \mathrm{eV}$, would entail no change in Fig. 2 because of the adoption of the universal index.

The comparison between quiescent and cosmic-ray matter distributions of Fig. 1 and 2 shows profound similarities and striking differences. Quiescent matter abundances of Fig. 1 are spread over 11 orders of magnitude while cosmic ray fluxes over only 3 orders of magnitude. The compression of the cosmic-ray matter distribution by 8 orders of magnitude in Fig. 2 is due to spallation reactions in the interstellar medium.

The Even-Odd Effect observed in the chemical composition of quiescent matter and cosmic ray is the major similarity and it suggests, or even demonstrates, that the composition of the cosmicray source matter in the interstellar medium is not overturned by any erratic mechanisms. The abundance hierarchy of $\mathrm{H}$ and $\mathrm{He}$ in quiescent matter also remains in cosmic-ray fluxes in the sense 
that $F_{c r}(H)>F_{c r}(H e)$ and $A_{g a}(H)>A_{g a}(H e)$ but the ratios $F_{c r}(H e) / F_{c r}(H)=0.81$ and $A_{g a}(H e) /$ $A_{g a}(H)=0.085$ are different.

The most notable differences in the two matter distributions regard secondary nuclei: $\mathrm{Li}, \mathrm{Be}$, and $\mathrm{B}$ and those close to the Iron group of elements, $\mathrm{Sc}, \mathrm{Ti}, \mathrm{Va}, \mathrm{Cr}$ and $\mathrm{Mn}$. As it is well known spallation reactions of primary nuclei with Hydrogen and Helium in the interstellar medium produce relative large amounts of secondaries. Since galactic abundances of $\mathrm{Li}, \mathrm{Be}$, and $\mathrm{B}$ are so scarce compared to Iron (respectively, $3.2 \times 10^{-3}, 5.6 \times 10^{-4}$ and $3.2 \times 10^{-6}$ ), small fractions of heavier parent nuclei suffering spallation suffice to produce significant amounts of $\mathrm{Li}, \mathrm{Be}$, and $\mathrm{B}$. This occurrence reduces the large abundance gaps of $L i, B e$ and $B$, in the range of $10^{-4}-10^{-6}$ in Fig. 1, to the milder gaps in the interval $10^{-1}-10^{-2}$ in Fig. 2.

Hydrogen and Helium are not affected by spallation reactions: the relative amounts of secondary protons and secondary He produced by spallation reactions in the interstellar medium do not upset the hierarchy $F_{c r}(H)>F_{c r}(H e)$ because the $H$ and $H e$ nuclei are too abundant compared to spallation production. This is evident from deuterium-to-proton flux ratio $\sim D / H \sim 5 \times 10^{-2}$ and that of the isotopes ${ }^{3} \mathrm{He} /{ }^{4} \mathrm{He} \sim(0.1-0.2)$, both measured at low energies [7] .

\section{Ratio of cosmic-ray to galactic abundances}

It is interesting to analyze the ratio of the two matter distributions. These quotients versus $\mathrm{Z}$ are shown in Fig. 3. Two main characteristics emerge from the sequence of ratios : (1) the EvenOdd Regularity exhibited by the data in Fig.s 1 and 2 is inverted; (2) the depletion of the elements is a strong, characteristic function of the atomic number in the range, $1 \leq \mathrm{Z} \leq 28$.

Generally spallation reactions enrich the odd nucleus (Z-1) with respect to the even $\mathrm{Z}$ nucleus in the range, $5 \leq Z \leq 28$ and hence the Even-Odd Effect observed in figure 1 and 2 is reversed in Fig. 3 : the even nucleus $\mathrm{Z}$ is underabundant with respect to the adjacent odd nucleus (Z-1). For example, in the case of the Boron-Carbon pair, the abundance ratios, $F_{c r}(B) / A_{g a}(B) \sim 3.5 \times 10^{3}$ and $F_{c r}(C) / A_{g a}(C) \sim 4 \times 10^{-2}$ exhibit the inverted regularity. Similarly it happens for the other 11 pairs in the range, $5 \leq \mathrm{Z} \leq 28$. It would not have been surprising if at least one of the twelve pairs (e.g. $B$ and $C, N$ and $O, F$ and $N e$ etc up to $C o$ and $N i$ ) would not have obeyed the inverted regularity ${ }^{1}$. The hierarchy in the $F_{c r} / A_{g a}$ quotients of the lightest nuclei, $1 \leq \mathrm{Z} \leq 2$ is not upset by spallation reactions because these elements are exceedingly abundant against spallation production as already noted.

The yellow strip in fig. 3 is a visual guide defined by an arbitrary factor \pm 2 around $F e=1$. All nuclei whose ratio $F_{c r} / A_{g a}$ is above the yellow band, are scarse as galactic abundances so that flux contributions via spallation reactions dominate the ratio, giving very high values $\sim 10^{5}$. All nuclei below the yellow strip are highly depleted at the injection stage of the Galactic accelerator.

$\mathrm{Fe}$ nuclei suffer spallation reactions generating sub-Iron species from $\mathrm{Sc}$ to $\mathrm{Mn}$. Iron refueling via spallation reactions from nuclei heavier than Nickel $(Z=28)$ is expected to be marginal on the basis that ultraheavy nucley are quite scarse and the fragment pseudorapidity distribution in nucleus-nucleus collisions is rather flat.

\footnotetext{
${ }^{1}$ Imagine a spiral galaxy with an average gas density in the interstellar medium significantly lower than 1 atom $/ \mathrm{cm}^{3}$ which is that of the Milky Way Galaxy. In this case the lower rates of spallation reactions would produce lower amounts of secondaries and some ratio could eventually conform with the inequality, $F_{c r}(Z-1) / A_{g a}((Z-1))<F_{c r}(Z) / A_{g a}((Z)$.
} 


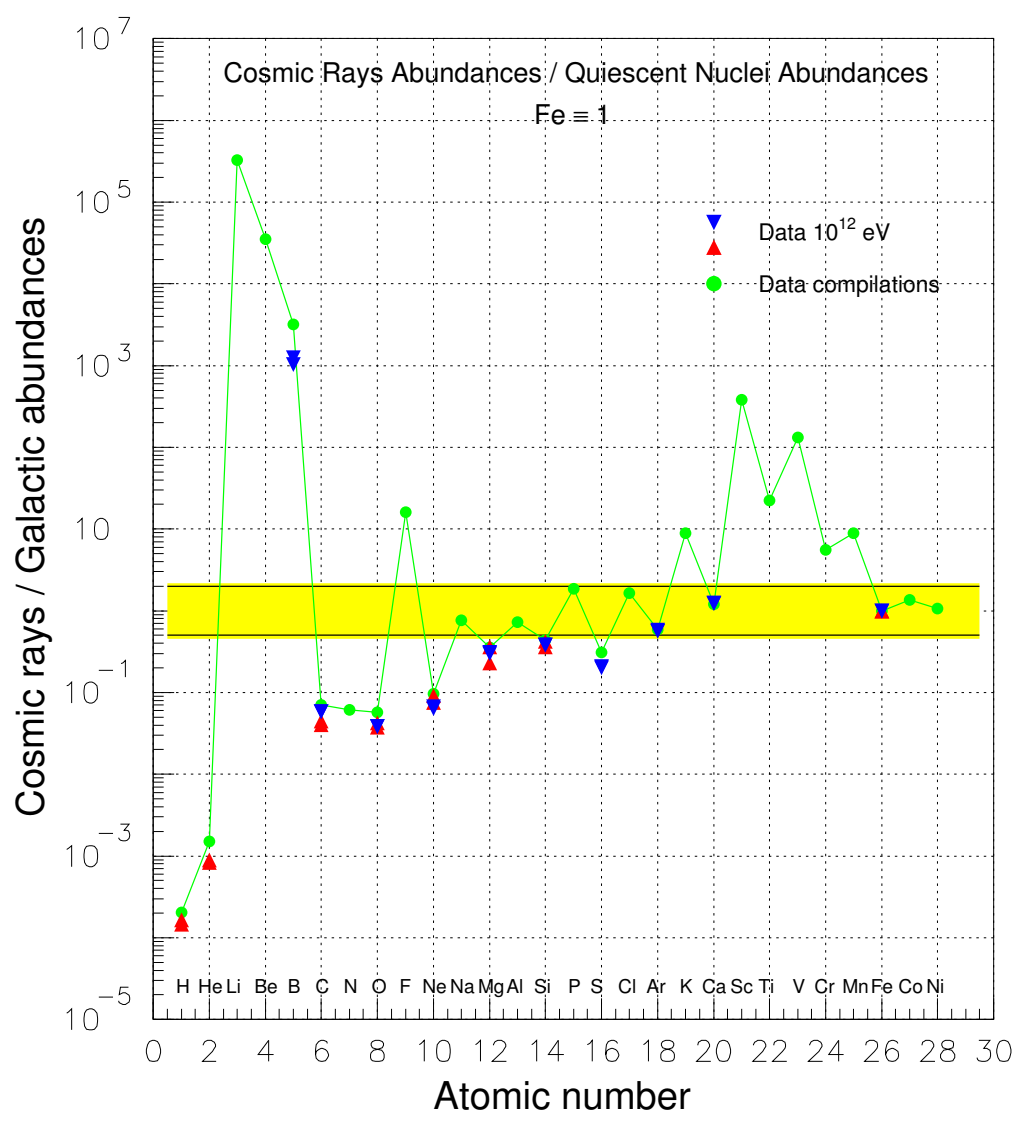

Figure 3: Ratios of cosmic-ray to quiescent matter abundances from Hydrogen to Nickel based on the data of Figures 1 and 2. The double triangles (hardly visible) translate the uncertainty in the extrapolation of the original data $[1,4]$ at $10^{12} \mathrm{eV}$.

In our opinion the Odd-Even and the Even-Odd effects are the major piece of empirical evidence that the injection phase of the Galactic accelerator takes place in the undifferentiated interstellar medium.

\section{Results and conclusions}

The universal phenomenon of spallation and the Odd-Even Effect exhibited by the data pattern in figure 3 pave the avenue for further analysis. These two effects on the abundance ratios $F_{c r}(Z) /$ $A_{g a}(Z)$ can be removed because they are qualitatively explained. Thus the analysis is restricted to a set of 11 even nuclei: $\mathrm{He}, \mathrm{C}, \mathrm{O}, \mathrm{Ne}, \mathrm{Mg}, \mathrm{Si}, \mathrm{S}, \mathrm{Ne}, \mathrm{Ca}, \mathrm{Fe}$ and $\mathrm{Ni}$ and the Hydrogen atom, the most abundant element.

The $F_{c r}(Z) / A_{g a}(Z)$ abundance ratios of the 12 nuclei are shown in figure 4 which are simply a subset of those shown in figure 3. A well definite pattern manifests itself in Fig. 4 marked by the pink curve. A simple parabolic interpolation through the 12 data points is given by the following 
equation :

$$
r=a_{1} Z^{2}-a_{2} Z
$$

where $a_{1}$ and $a_{2}$ are two numerical constants, $a_{1}=1.31 \times 10^{-3}$ and $a_{2}=9.65 \times 10^{-4}$. The rule has a simple physical meaning : nuclei of the quiescent matter in cosmic-ray sources, before undergoing acceleration, suffer a filtering process which depends on the atomic number $Z$ according to the equation (1). Higher is the atomic number $Z$ lower is the sieve effect: accordingly Hydrogen is the most sieved atom while $\mathrm{Fe}$ and Ni nuclei easily permeate the cosmic-ray filter at the source.

The effect of the filter on the cosmic-ray source matter is gigantic: $\mathrm{H}$ and $\mathrm{Fe}$ are disequalized by a factor $\sim 10^{4}$. The enormity of the sieve is undeniable and it escaped any previous analyses. The abundance ratios of cosmic-ray fluxes expressed in $\mathrm{GeV} / \mathrm{n}$ are routinely reported in the literature since decades. In this unit of measurements data points do not aggregate around the smooth, regular curve as that in Fig. 4 (pink profile) but they are erratically scattered.

The importance of the new result expressed by the equation (1) can be appreciated in comparison of previous analyses aiming at to ascertain a simple rule governing the abundance ratios. For example it was conjectured a preferential leakage of partially ionized nuclei in the source, just before the acceleration [8] . Such a leakage would depend on the M/Q ratio where $M$ is the mass and Q the effective charge of the particle. This conjecture would explain the moderate abundance of heavy nuclei with respect to light nuclei in Fig. 3.

Another attempt [9] in 1971 related the abundance ratios of Fig. 3 to the first ionization potential $I(Z)$ of the elements (anticorrelation) . This conjecture was successively analyzed [10] in various contexts. The proposed anticorrelation is qualitatively correct in the sense that heavy elements are more abundant that light elements according to the data of Fig. 3. Quantitatively the anticorrelation is brutally violated for Helium, being $\left[F_{c r}(H) / F_{c r}(F e)\right] /\left[A_{g a}(H) / A_{g a}(F e)\right] \ll[$ $\left.F_{c r}(\mathrm{He}) / F_{g a}(\mathrm{Fe})\right] /\left[A_{g a}(\mathrm{He}) / A_{g a}(\mathrm{Fe})\right]$ and $\mathrm{I}(\mathrm{H})<I(\mathrm{He})$. Hence, in strict terms, the claim for the global anticorrelation is fictitious.

It is a fact that volatile elements $(\mathrm{H}, \mathrm{He}, \mathrm{C}, \mathrm{N}, \mathrm{O}, \mathrm{Ne}$ and $\mathrm{Ar}$ with condensation temperatures $\leq$ $800 \mathrm{~K}$ ) do have high ionization potentials, and accordingly, it is plausible to conjecture that cosmicray source matter populates galactic zones where volatile elements are depleted. This possibility has been explored with many variants by a number of authors. All elements have been differentiated in high volatile, volatile, semivolatile, refractory $(\mathrm{Mg}, \mathrm{Al}, \mathrm{Si}, \mathrm{Ca}, \mathrm{Fe}, \mathrm{Ni}$ and $\mathrm{Co}$ with condensation temperatures $\leq 1240 \mathrm{~K}$ ), and other categories.

But the general pattern of the abundance ratios shown in Fig. 3 cannot be explained with the first ionization potential or the volatility concept. For example, elements such as S and Zn have different abundance ratios but comparable ionization potentials and comparable volatilities.

The chemical composition of cosmic rays at the sources resembles to that of cosmic rays emitted in solar flares : both depend on the ionization degree of the atoms [11, 12]. Yet this resemblance does not explain the data pattern in Fig. 3. All these attempts are plagued either by vagueness or by many exceptions if a definite rule between abundance ratio and a specific variable is imposed.

Those who are familiar with the Principle of Constant Indices $(P C I)$ will recognize in the new result, the rule without exceptions expressed by the equation (1), a real ordo rerum in the data. For those who ignore the PCI the new result is a simple change of variable from electronvolt 


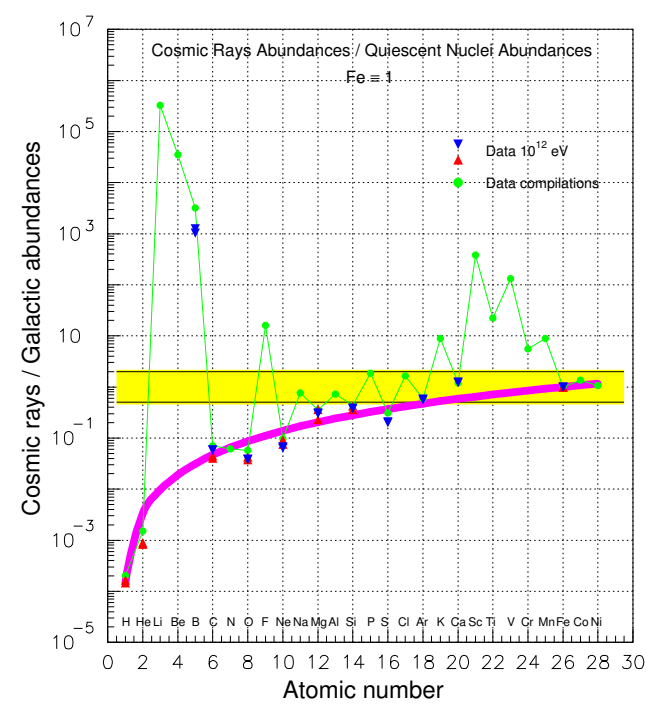

Figure 4: Evidence for the parabolic rule (pink curve) obeyed by even nuclei and Hydrogen in the range $1 \leq \mathrm{Z} \leq 28$.

per nucleon to electronvolt per particle. In both cases the rule is an inescapable starting point to identify the physical process sieving cosmic rays before acceleration.

\section{References}

[1] A. Obermeyer et al., (Tracer Coll.) 2011, arXiv: 1108.4838v1, 24 Aug. 2011.

[2] M. Asplumb, N. Grevesse et al., 2009, arXiv: 0909.0948v1, 4 Sept. 2009.

[3] E. Anders and N. Grevesse 1989, Geochim. Cosmochim. Acta, 53 pages 197-214.

[4] H. S. Ahn et al. (Cream Coll.) 2008, arXiv: 0808.1718v1, 12 Aug. 2008.

[5] Wiebel Sooth et al. 2009, arXiv: 0909.0948v1, 4 Sept. 2009.

[6] A. Codino (2013), Progress and Prejudice in Cosmic Ray Physics until 2006, Societá Editrice Esculapio, Bologna, Italy (http://www.editrice-esculapio.com/codino-progress-and-prejudice-incosmic-ray-physics-until-2006/).

[7] W. R. Weber and Yushak (1983), The Astrophys. Journal, 275, 391.

[8] P. B. Price et al. (1971) Phys. Rev. Letters, 26, 916.

[9] O. Havnes, 1971, Nature, 229, 548.

[10] M. Cassé and P. Goret (1978) The Astrophys. Journal, 221, 703.

[11] W. R. Binns et al., (1981) The Astrophys. Journal, 247, L115.

[12] W. R. Webber (1975) Proc. 14 ICRC, 1597, Munich, Germany. 